\title{
ON SCHUR-CONVEXITY AND SCHUR-GEOMETRIC CONVEXITY OF FOUR-PARAMETER FAMILY OF MEANS
}

\section{ALFRED WITKOWSKI}

Abstract. We prove that the four-parameter family of means

$$
R(u, v ; r, s ; x, y)=\left[\frac{E\left(r, s ; x^{u}, y^{u}\right.}{E\left(r, s ; x^{v}, y^{v}\right)}\right]^{1 /(u-v)}
$$

is Schur-geometrically convex (concave) in $x, y$ if $(u+v)(r+s) \geqslant(\leqslant) 0$, and Schur-concave (convex) in $u, v \geqslant 0$ if $r+s \geqslant(\leqslant) 0$.

Mathematics subject classification (2010): 26B25.

Keywords and phrases: S-means, Gini mean, Stolarsky mean, Schur-convexity, Schur-geometric convexity.

\section{REFERENCES}

[1] P.S. Bullen, Handbook of Means and their Inequalities, Kluwer Academic Press, Dordrecht, 2003.

[2] Y. Chu, X. Zhang And G. WANG, The Schur Geometrical Convexity of the Extended Mean Values, Journal of Convex Analysis 15 (2008), 707-718.

[3] A.W. Marshall AND I. Olkin, Inequalities: Theory of Majorization and Its Applications, Academic Press, New York, 1979.

[4] F. QI, A note on Schur-convexity of extended mean values, Rocky Mountain J.Math. 35, 5 (2005), 1787 1793.

[5] J. SÁNDOR, The Schur-convexity of Stolarky and Gini means, Banach J. Math. Anal. 1, 2 (2007), 212 215.

[6] H.-N. SHI, Y.-M. JIANG AND W.-D. JIANG, Schur-convexity and Schur-geometrically concavity of Gini means, Comput. Math. Appl. 57, 2 (2009), 266-274.

[7] K.B. StOlars Ky, Generalizations of the logarithmic mean, Math. Mag. 48 (1975), 87-92.

[8] A. WitkowsKi, Comparison theorem for two-parameter means, Math. Inequal. Appl. 12, 1 (2009), $11-20$.

[9] A. WitkowsKi, Monotonicity and convexity of S-means, Math. Inequal. Appl, 13, 1 (2010), 33-42. 